\title{
PLK1 Inhibitor CYC140
}

National Cancer Institute

\section{Source}

National Cancer Institute. PLK1 Inhibitor CYC140. NCI Thesaurus. Code C162375.

A competitive inhibitor for adenosine triphosphate (ATP) binding to polo-like kinase 1

(PLK1; PLK-1; STPK13), with potential antineoplastic activity. Upon administration, PLK1

inhibitor CYC140 selectively targets, binds to and inhibits PLK1, which disrupts mitosis and induces selective G2/M cell-cycle arrest followed by apoptosis in PLK1-overexpressing tumor cells. PLK1, named after the polo gene of Drosophila melanogaster, is a serine/threonine kinase that is crucial for the regulation of mitosis, and plays a key role in tumor cell proliferation, transformation and invasion. PLK1 expression is upregulated in a variety of tumor cell types and high expression is associated with increased aggressiveness and poor prognosis. 